\title{
Existence of traveling waves for integral recursions with nonmonotone growth functions
}

\author{
Bingtuan Li * \\ Department of Mathematics \\ University of Louisville \\ Louisville, KY 40292 \\ Mark A. Lewis ${ }^{\dagger}$ \\ Department of Mathematical and Statistical Sciences \\ Department of Biological Sciences \\ CAB 632 University of Alberta \\ T6G 2G1 Canada \\ mlewis@math.ualberta.ca \\ 780-492-0197 (voice) 780-492-6826 (fax) \\ Hans F. Weinberger \\ School of Mathematics \\ University of Minnesota \\ 206 Church Street S.E. \\ Minneapolis, Minnesota 55455 \\ Journal of Mathematical Biology 58 (2009), 323-338, \\ DOI:10.1007/s00285-008-0175-1
}


Short Title: Traveling waves in overcompensated integral recursions

Keywords: traveling waves, nonmonotone growth function, spreading speed, integral recursion

AMS Classification: 92D40, 92D25

*This research was partially supported by the National Science Foundation under Grant DMS-616445.

†The Canada Research Chairs program, and a grant from the Natural Sciences and Engineering Research Council of Canada. 


\begin{abstract}
A class of integral recursion models for the growth and spread of a synchronized single-species population is studied. It is well known that if there is no overcompensation in the fecundity function, the recursion has an asymptotic spreading speed $c^{*}$, and that this speed can be characterized as the speed of the slowest non-constant traveling wave solution. A class of integral recursions with overcompensation which still have asymptotic spreading speeds can be found by using the ideas introduced by Thieme [10] for the study of space-time integral equation models for epidemics. The present work gives a large subclass of these models with overcompensation for which the spreading speed can still be characterized as the slowest speed of a non-constant traveling wave. To illustrate our results, we numerically simulate a series of traveling waves. The simulations indicate that, depending on the properties of the fecundity function, the tails of the waves may approach the carrying capacity monotonically, may approach the carrying capacity in an oscillatory manner, or may oscillate continually about the carrying capacity, with its values bounded above and below by computable positive numbers.
\end{abstract}

\title{
1 Introduction.
}

The growth and spread of a synchronized single-species population is often modeled by an integral recursion of the form

$$
u_{n+1}(x)=Q\left[u_{n}\right](x):=\int_{-\infty}^{+\infty} k(x-y) f\left(u_{n}(y)\right) d y
$$

Here $u_{n}(x)$ is the density of individuals at point $x$ and time $n, f(u)$ describes the density-dependent fecundity, and $k(x-y)$ is the dispersal function, which depends upon the signed distance $x-y$ between the location of birth $y$ and the location of settlement $x$. The recursion (1.1) describes the reproduction and dispersal of a time-synchronized species in which all individuals first undergo reproduction and then redistribute their offspring before reproduction occurs once again. This is a good model for annual plants and many insect species.

It is well-known (see, e.g. $[12,13])$ that if $f(u)$ is nondecreasing, then the integral recursion (1.1) has a forward asymptotic spreading speed $c^{*}$, and $c^{*}$ can be characterized as the slowest speed of a family of non-constant traveling wave solutions of (1.1). Moreover it is shown that if fecundity has the additional property that $f(u) \leq f^{\prime}(0) u$, then $c^{*}$ can be found from a simple formula which depends only on $f^{\prime}(0)$ and the migration kernel $k(x)$. 
Horst Thieme [9] pointed out that the latter result can be formally obtained from his results on the spreading speed under a continuous-time integrodifferential equation model by requiring the birth-migration kernel $k(t, x)$ of this model to take the form $\delta(t-1) k(x)$ with $\delta$ the Dirac distribution and $k(x)$ the dispersal function of equation (1.1)).

It is known that fecundity functions $f(u)$ may not be nondecreasing. This is, for instance, true of the Ricker function $f(u)=u e^{r-u}$ with $r>1$, and of the logistic function $f(u)=u[1+r(1-u)]$ with $0<r<3$. If $f(u)$ decreases on some range of $u$, the phenomenon of overcompensation is said to occur.

In [10] Thieme showed that a spreading result can still be obtained for space-time integral equations under assumptions about the nonlinearity $f$ which imply that $f(u) \leq f^{\prime}(0) u$ but permit $f$ to be the Ricker or logistic function, and that the number $c^{*}$ given by the above-mentioned formula is, in fact, the spreading speed. Proposition 3.1 will give a more precise statement of the analogous result for the recursion (1.1). The purpose of the present work is to show that under some assumptions which are slightly stronger than those of [10] but which are satisfied by the Ricker and logistic functions, the speed $c^{*}$ can, as in $[12,13]$, be characterized as the speed of the slowest member of a family of non-constant traveling wave solutions of (1.1). That is, we shall show that for every $c \geq c^{*}$ there is a nonnegative solution of the form $u_{n}(x)=w(x-n c)$ of (1.1) with $w(\infty)=0$ but $w$ bounded away from zero near $-\infty$, and that there is no such wave when $0 \leq c<c^{*}$.

The problem will be formulated in Section 2 and 3. Our main result will be stated and proved in Section 4. Section 5 shows numerical simulations of the graphs of some of these waves. Section 6 will discuss some relations of our results to other work. In particular, it will be shown that our theorem on the one-space-dimensional recursion (1.1) can immediately be applied to finding the traveling waves of a large class of integral recursion and integral equation models in any number of dimensions.

\section{Hypotheses and definitions.}

We shall make the following assumptions, the first two of which are satisfied by the Ricker function $f(u)=u e^{r-u}$ when $r>0$.

\section{Hypotheses 2.1.}

$i$. There is a positive constant $\alpha_{0}$ such that 

a. $f(u)$ is continuous for $0 \leq u \leq \alpha_{0}$;
b. $0<f(u) \leq \alpha_{0}$ for $0<u \leq \alpha_{0}$; and
c. $f(0)=0$.

ii. There is a positive constant $d \leq \alpha_{0}$ such that

a. $f(u)$ is nondecreasing for $0<u \leq d$;

$b$. the specific net growth rate $f(u) / u$ is bounded and nonincreasing for $0<u \leq d$;

c. $f(u) / u>1$ for $0<u<d$; and

d. $f(u) / u \leq f(d) / d$ for $d \leq u \leq \alpha_{0}$.

iii. $k(x)$ is a continuous and nonnegative function such that

a. $\int_{-\infty}^{+\infty} k(x) d x=1$;

b. the integral

$$
K(\mu):=\int_{-\infty}^{\infty} k(x) e^{\mu x} d x
$$

is finite for at least one positive and one negative value of $\mu$.

Remark. Hypotheses 2.1.ii.b, c, and d imply that the function $f$ is right differentiable at 0 , that $f^{\prime}(0)>1$, and that $f(u) \leq f^{\prime}(0) u$ for $0 \leq u \leq \alpha_{0}$.

Define the function

$$
f^{+}(u):=\max _{0 \leq v \leq u} f(v)
$$

for $0 \leq u \leq \alpha_{0}$. (This function is denoted by $G(u, 0)$ in [10].) $f^{+}(u)$ is a continuous and nondecreasing function, and $0 \leq f(u) \leq f^{+}(u) \leq \alpha_{0}$ for $0 \leq u \leq \alpha_{0}$. Note that Hypothesis 2.1.ii.a shows that $f^{+}(u)=f(u)$ for $u \leq d$.

Since Hypothesis 2.1.ii.c shows that $f^{+}(u)>u$ for $0<u<d$, and since $f^{+}\left(\alpha_{0}\right) \leq \alpha_{0}$ by Hypothesis 2.1.i.b, there must be at least one root of the equilibrium equation $f^{+}(u)=u$ on the interval $\left[d, \alpha_{0}\right]$, and there are no such roots in the interval $(0, d)$. We define $\alpha$ to be the smallest positive solution of the equilibrium equation $f^{+}(u)=u$. Then

$$
f(\alpha)=\alpha, \text { and } f(u)>u \text { for } 0<u<\alpha .
$$

We now define the function

$$
f^{-}(u):=\min _{u \leq v \leq \alpha} f(v) \text { for } 0 \leq u \leq \alpha .
$$


(This function is called $G(u, \alpha)$ in [10].) Clearly, $f^{-}(u) \leq f(u)$. In fact, $f^{-}$ is the largest nondecreasing function which lies below $f$ on the interval $[0, \alpha]$. Because $f$ is nondecreasing on the interval $[0, d]$, we obtain the formula

$$
f^{-}(u)=\min \left\{f(u), f^{-}(d)\right\} \text { when } 0 \leq u \leq d .
$$

This and Hypothesis 2.1.ii.c show that $f^{-}(u)=f(u)>u$ for all sufficiently small positive $u$. Since $f^{-}(\alpha) \leq f^{+}(\alpha)=\alpha$, there is a smallest positive solution $\sigma$ of the equation $f^{-}(u)=u$. This constant has the properties

$$
0<\sigma \leq u^{*} \leq \alpha, f^{-}(\sigma)=\sigma, \text { and } f^{-}(u)>u \text { for } 0<u<\sigma
$$

where $u^{*}$ is defined to be the smallest positive root of the equation $f(u)=u$. $u^{*}$ is called the carrying capacity of the system defined by the recursion (1.1).

\section{The spreading speed}

An approach parallel to the one in [10] can be used to obtain a spreading speed $c^{*}$ for the recursion (1.1) .

Proposition 3.1. Suppose that the Hypotheses 2.1 are satisfied. Define the numbers

$$
c^{*}:=\inf _{\mu>0}\left[(1 / \mu) \ln \left\{\int_{-\infty}^{\infty} e^{\mu y} k(y) d y\right\}\right]
$$

and

$$
c^{*}(-1):=\inf _{\mu>0}\left[(1 / \mu) \ln \left\{\int_{-\infty}^{\infty} e^{-\mu y} k(y) d y\right\}\right] .
$$

Then $c^{*}$ is the asymptotic rightward spreading speed of the recursion (1.1) in following sense:

If the continuous initial function $u_{0}(x)$ is zero for all sufficiently large $x$, $u_{0} \not \equiv 0$, and $0 \leq u_{0}(x) \leq \alpha$, then for any positive $\epsilon$ the solution of $u_{n}$ of the integral recursion (1.1) has the following properties

$i$.

$$
u_{n}(x) \leq \alpha \text { for all } x \text { and } n .
$$

ii.

$$
\lim _{n \rightarrow \infty}\left[\sup _{x \geq n\left(c^{*}+\epsilon\right)} u_{n}(x)\right]=0
$$


iii. $c^{*}+c^{*}(-1)>0$, and

$$
\liminf _{n \rightarrow \infty}\left[\inf _{-n\left(c^{*}(-1)-\epsilon\right) \leq x \leq n\left(c^{*}-\epsilon\right)} u_{n}(x)\right] \geq \sigma .
$$

The proof of this Proposition is based on the well-known result:

Proposition 3.2. Comparison Principle. Let $R[u]$ be an operator which takes the set of nonnegative bounded continuous functions $u(x)$ into functions in the same set, and which is monotone in the sense that $u \geq v$ implies that $R[u] \geq R[v]$. If $\left\{v_{n}\right\}$ and $\left\{w_{n}\right\}$ are two sequences with the properties $v_{n+1} \leq R\left[v_{n}\right]$ and $w_{n+1} \geq R\left[w_{n}\right]$ for all nonnegative $n$, and if $v_{0}(x) \leq w_{0}(x)$, then $v_{n} \leq w_{n}$ for all positive $n$.

This property is proved by induction.

The proof of Proposition 3.1 makes use of the two auxiliary operators

$$
Q^{+}[u](x):=\int_{-\infty}^{+\infty} k(x-y) f^{+}(u(y)) d y
$$

and

$$
Q^{-}[u](x):=\int_{-\infty}^{+\infty} k(x-y) f^{-}(u(y)) d y .
$$

We also define $Q[u]$ to be the integral operator on the right of (1.1). By construction, $0 \leq f^{-}(u) \leq f(u) \leq f^{+}(u) \leq \alpha$ for $0 \leq u \leq \alpha$, and hence

$$
Q^{-}[u](x) \leq Q[u](x) \leq Q^{+}[u](x)
$$

for all nonnegative continuous functions $u$ with $u \leq \alpha$. The Comparison Principle shows that if $u_{n}^{+}$is a solution of the recursion

$$
u_{n+1}^{+}=Q^{+}\left[u_{n}^{+}\right]
$$

$\left\{u_{n}^{-}\right\}$is a solution of the recursion

$$
u_{n+1}^{-}=Q^{-}\left[u_{n}^{-}\right]
$$

and $\left\{u_{n}\right\}$ is a solution of the recursion (1.1), and if $0 \leq u_{0}^{-}(x) \leq u_{0}(x) \leq$ $u^{+}(x) \leq \alpha$, then

$$
0 \leq u_{n}^{-}(x) \leq u_{n}(x) \leq u_{n}^{+}(x) \leq \alpha
$$

for all $x$ and $n$. Because $f^{+}$and $f^{-}$are nondecreasing and have the same derivative at zero, the results of [12] show that the recursions for $u^{+}$and $u^{-}$ 
have the spreading speed (3.1). In particular, the statement (3.3) is valid with $u$ replaced by $u^{+}$, and the statement (3.4) is valid with $u$ replaced by $u^{-}$. In order to finish the proof, we only need to show that $c^{*}+c^{*}(-1)>0$. For this purpose, we use Schwarz's inequality to see that the logarithms in the definitions (3.1) and (3.2) of $c^{*}$ and $c^{*}(-1)$ are strictly convex in $\mu$. (For an equivalent derivation, see the definitions (9.4) and (9.6) and the equation (9.7) of [13] with $\xi=1$.)

Convexity shows that the logarithm on the right of (3.2) is bounded below by its linearization at 0 , and this implies that $c^{*}(-1)$ is bounded below by the derivative at $\mu=0$ of this logarithm. A simple calculation shows that this derivative is the negative of the derivative of the logarithm in (3.1) at $\mu=0$. Therefore, we can obtain a lower bound for $c^{*}+c^{*}(-1)$ by replacing the logarithm on the right of (3.1) by this logarithm minus $\mu$ times its derivative at zero. Thus, a lower bound for $c^{*}+c^{*}(-1)$ is obtained by finding the infimum of $1 / \mu$ times a strictly convex function which is positive and has the derivative 0 at 0 . It is easily seen that this infimum is bounded below by the (positive) slope of any tangent line to the graph which lies above the $\mu$-axis at $\mu=0$. This shows that $c^{*}+c^{*}(-1)>0$. We have thus established all the statements of Proposition 3.1.

Remarks. 1. The same proof shows that $c^{*}(-1)$ is the leftward asymptotic spreading speed of (1.1).

2. The statement (3.3) shows that for any positive $\epsilon$, all points of any level set $\left\{x: u_{n}(x)\right\}=\eta$ with $\eta<\alpha$ must lie in the interval $x \leq n\left(c^{*}+\epsilon\right)$ when $n$ is sufficiently large. The statement (3.4) shows that for all sufficiently large $n$ the interval $x \geq n\left(c^{*}-\epsilon\right)$ contains points of the above level set with $\eta<\sigma$, but permits level sets with $\eta>\sigma$ to spread more slowly or not at all.

\section{The existence of traveling waves.}

A solution of the recursion (1.1) is said to be a traveling wave of speed $c$ if it has the form $u_{n}(x)=w(x-n c)$, where $w$ is a function of one variable. By substituting this form into the recursion, we see that $w$ is a traveling wave if and only if it satisfies the equation

$$
w(x)=Q_{c}[w](x),
$$

where we have defined the operator

$$
Q_{c}[w](x):=Q[w](x+c)=\int_{-\infty}^{\infty} f(w(y)) k(x+c-y) d y .
$$


Thus, a traveling wave is a fixed point of the operator $Q_{c}$. In a similar manner, we can define traveling waves of the recursions (3.5) and (3.6) as fixed points of the operators $Q_{c}^{+}[u](x):=Q^{+}[u](x+c)$ and $Q_{c}^{-}[u](x):=$ $Q^{-}[u](x+c)$, respectively. Because the operators $Q^{ \pm}$are order-preserving, the results of $[12,13]$, show that for all $c \geq c^{*}$, the integral recursions $(3.5)$ and (3.6) have nontrivial nonincreasing traveling wave solutions $w^{+}(x-n c)$ and $w^{-}(x-n c)$, respectively, with $w^{ \pm}(+\infty)=0, w^{+}(-\infty)=\alpha$, and $w^{-}(-\infty)=$ $\sigma$. In this section we shall show that the recursion (1.1) also has non-constant traveling wave solutions of every speed $c \geq c^{*}$.

Our main result is the following theorem, which shows that $c^{*}$ can be characterized as the slowest speed of a class of traveling wave solutions for the recursion (1.1).

Theorem 4.1. For any $c \geq c^{*}$, the integral recursion (1.1) has a traveling wave solution $u_{n}(x)=w(x-n c)$ with $w(x) \leq \alpha$ for all $x, w(+\infty)=0$, and $\liminf \operatorname{in}_{x \rightarrow-\infty} w(x) \geq \sigma$. A traveling wave solution $w(x-n c)$ with $w(\infty)=0$ and $\lim \inf _{x \rightarrow-\infty} w(x)>0$ does not exist if $c<c^{*}$.

We shall use three lemmas in the proof of the Theorem.

Lemma 4.1. The number

$$
\ell:=\sup \left\{u: 0<u<d \text { and } f(u)<f^{-}(d)\right\}
$$

satisfies the inequalities $0<\ell \leq d$, and

$$
f^{-}(u)=f^{+}(u)=f(u) \text { for } 0 \leq u \leq \ell
$$

Proof. Because $f$ is continuous and strictly positive for $u>0, f^{-}(d)>0$. Since $f(0)=0, f(u)<f^{-}(d)$ for all sufficiently small $u$, so that $\ell>0$. Because $f(d) \geq f^{-}(d), \ell \leq d$. The formula (2.5) shows that $f^{-}(u)=f(u)$ for $u \leq \ell$. The definition (2.2) shows that $f^{+}(u)=f(u)$ for $u \leq d$, and hence for $u \leq \ell$. This finishes the proof of the Lemma.

Lemma 4.2. If $0<\gamma<\ell / \alpha$ where $\ell \leq d$ is defined by (4.2), and if $0<u \leq$ $\alpha$, then $f^{-}(\gamma u) \geq \gamma f^{+}(u)$.

Proof. Since $\gamma u \leq d$, Hypothesis 2.1.ii.a shows that

$$
f(v) \leq f(\gamma u) \text { for } 0 \leq v \leq \gamma u
$$


On the other hand, Hypothesis 2.1.ii.b shows that

$$
f(v) \leq v f(\gamma u) /(\gamma u) \text { for } \gamma u \leq v \leq d
$$

and Hypothesis 2.1.ii.d then yields the same inequality for $d \leq v \leq \alpha$. The definition (2.2) of $f^{+}$and Lemma 4.1 show that

$$
f^{+}(u) \leq f(\gamma u) / \gamma=f^{-}(\gamma u) / \gamma
$$

This is the statement of the Lemma.

We shall use the usual Banach space $C(-\infty, \infty)$ of bounded continuous functions with the maximum norm $\|u\|=\sup _{x \in(-\infty,+\infty)}|u(x)|$. For any $c \geq c^{*}$, let

$$
\begin{array}{r}
E_{c}=\{u(x): u \text { in } C(-\infty, \infty), \\
\left.\gamma w^{+}(c ; x) \leq u(x) \leq w^{+}(c ; x)\right\} \\
\text { with } 0<\gamma<\min \{\sigma, \ell\} / \alpha .
\end{array}
$$

Clearly $E_{c}$ is a bounded nonempty closed convex subset of $C(-\infty, \infty)$.

Lemma 4.3. The operator

$$
Q_{c}[u](x):=Q[u](x+c)=\int_{-\infty}^{\infty} k(x+c-y) f(u(y)) d y
$$

maps $E_{c}$ into $E_{c}$.

Proof. Define the operators

$$
Q_{c}^{ \pm}[u]=\int_{-\infty} k(x+c-y) f^{ \pm}(u(y)) d y
$$

so that $Q_{c}^{+}\left[w^{+}\right]=w^{+}$. Because $f^{+} \geq f$ and $f^{+}$is nondecreasing, we see that if $u \leq w^{+}$, then

$$
Q_{c}[u] \leq Q_{c}^{+}[u] \leq Q_{c}^{+}\left[w^{+}\right]=w^{+} .
$$

On the other hand, Lemma 4.2 shows that if $u \geq \gamma w^{+}$, then

$$
Q_{c}[u] \geq Q_{c}^{-}[u] \geq Q_{c}^{-}\left[\gamma w^{+}\right] \geq \gamma Q_{c}^{+}\left[w^{+}\right]=\gamma w^{+}
$$

Thus if $u$ is in $E_{c}$, the same is true of $Q_{c}[u]$. This is the statement of the Lemma.

We are now ready to prove Theorem 4.1.

\section{Proof of Theorem 4.1.}


To show the first statement of the Theorem, we define the operators

$$
Q_{c}^{(m)}[u](x)=\left\{\begin{array}{cc}
Q_{c}[u](x), & \text { if } x \geq-m \\
\max \left\{Q_{c}[u](-m), \gamma w^{+}(c ; x)\right\}, & \text { if } x \leq-m
\end{array}\right.
$$

for every positive integer $m$ and all $u$ in $E_{c}$. In view of Lemma $4.3, Q_{c}^{(m)}$ takes $E_{c}$ into $E_{c}$.

By multiplying the continuous function $k(x)$ by a suitable cutoff function, one can, for any positive $\epsilon$, find a continuous function $k_{\epsilon}(x)$ which vanishes outside a bounded interval $\left[-M_{\epsilon}, M_{\epsilon}\right]$ and satisfies the inequality

$$
\int_{-\infty}^{\infty}\left|k_{\epsilon}-k\right| d x<\epsilon / 3
$$

Because $k_{\epsilon}$ is uniformly continuous, $\left|k_{\epsilon}(x+\eta)-k_{\epsilon}(x)\right|<\epsilon /\left\{6\left[1+M_{\epsilon}\right]\right\}$ for all sufficiently small $\eta$. Since $\epsilon$ is arbitrary, it follows that

$$
\lim _{\eta \rightarrow 0} \int_{-\infty}^{\infty}|k(x+\eta)-k(x)| d x=0 .
$$

This implies that the family $Q_{c}[u]$ with $0 \leq u \leq \alpha$ is equicontinuous. On the other hand, $w^{+}$is uniformly continuous, and hence the family $Q_{c}^{(m)}[u]$ is also equicontinuous. Then Ascoli's theorem implies that every sequence $v_{n}(x)$ in $E_{c}$ has a subsequence $v_{n_{\kappa}}(x)$ such that $Q_{c}^{(m)}\left[v_{n_{\kappa}}\right](x)$ converges to a function $s(x)$ uniformly on every bounded interval. We wish to show that $Q_{c}^{(m)}\left[v_{n_{\kappa}}\right](x)$ converges to $s(x)$ uniformly on the whole real line $(-\infty,+\infty)$. For this purpose we observe that for any given $\epsilon>0$, there exist $M>-m$ such that $w^{+}(c ; M)<\epsilon$, and $N>0$ such that for $\kappa \geq N$, $\sup _{-m \leq x \leq M} \mid Q_{c}\left[v_{n_{\kappa}}\right](x)-$ $s(x) \mid<\epsilon$. Because both $Q_{c}\left[v_{n_{\kappa}}\right]$ and $s$ are nonnegative and bounded above by the nonincreasing function $w^{+}(c ; x)$, we see that

$$
\sup _{x \geq M}\left|Q_{c}^{(m)}\left[v_{n_{\kappa}}\right](x)-s(x)\right| \leq w^{+}(c ; M)<\epsilon \text { when } \kappa>N .
$$

Some simple arithmetic shows that when $x \leq-m$ and $\kappa>N$,

$$
\begin{aligned}
\left|Q_{c}^{(m)}\left[v_{n_{\kappa}}\right](x)-s(x)\right| & =\mid \max \left\{Q_{c}\left[v_{n_{\kappa}}\right](-m), \gamma w^{+}(c ; x)\right\} \\
& -\max \left\{s(-m), \gamma w^{+}(c ; x)\right\} \mid \\
\leq & \left|Q_{c}\left[v_{n_{\kappa}}\right](-m)-s(-m)\right|<\epsilon .
\end{aligned}
$$

Since $\left|Q_{c}^{(m)}\left[v_{n_{\kappa}}\right](x)-s(x)\right| \leq \epsilon$ for $-m \leq x \leq M$ when $k \geq N$, we have shown that $Q_{c}^{(m)}\left[v_{n_{\kappa}}\right]$ converges to $s(x)$ uniformly on the whole real line. That is, $Q_{c}^{(m)}$ takes the convex set $E_{c}$ into a compact subset of $E_{c}$. The 
Schauder Fixed Point Theorem (see, e. g., [2], pp. 403-406) shows that there exists $w^{(m)}(x) \in E_{c}$ such that $Q_{c}^{(m)}\left[w^{(m)}\right]=w^{(m)}$. Because the family $w^{(m)}$ is again equicontinuous, there is a sequence $m_{i} \rightarrow \infty$ as $i \rightarrow \infty$ such that $Q_{c}^{\left(m_{i}\right)}\left[w^{\left(m_{i}\right)}\right](x)$ converges to a function $w(x) \in E_{c}$ uniformly for $x$ on every bounded interval. It follows that $w^{\left(m_{i}\right)}(x)$ converges to $w(x)$ uniformly for $x$ on every bounded interval. This, Hypothesis 2.1.iii.a, and the definition of $Q_{c}^{(m)}$ show that $Q_{c}^{\left(m_{i}\right)}\left[w^{\left(m_{i}\right)}\right](x) \rightarrow Q_{c}[w](x)$ for all $x$ as $i \rightarrow \infty$. We therefore have $Q_{c}[w](x)=w(x)$ for all $x$. It follows that $Q[w(\cdot-n c)](x)=w(x-(n+$ $1) c)$, so that $w$ is a traveling wave solution of (1.1).

We have shown that for any $c \geq c^{*}$ the recursion (1.1) has a traveling wave solution $w(x-n c)$ with $\gamma w^{+}(c ; x) \leq w(x) \leq w^{+}(c ; x)$ for all $x$. Since $w^{+}(c ; x) \leq \alpha$ for all $x$ and $w^{+}(c ; \infty)=0$, we have $w(c ; x) \leq \alpha$ for all $x$, and $w(\infty)=0$.

To obtain the behavior of $w$ at $-\infty$, we consider the recursion

$$
u_{n+1}^{-}=Q_{c}^{-}\left[u_{n}^{-}\right]
$$

with the initial condition

$$
u_{0}^{-}=\gamma w^{+} .
$$

Lemma 4.2 shows that $Q_{c}^{-}\left[u_{0}^{-}\right] \geq u_{0}^{-}$, and it follows by induction that $u_{n}^{-}$is nondecreasing in $n$ and nonincreasing in $x$. Moreover, since $Q_{c}^{-}[w] \leq Q_{c}[w]=$ $w$ and $u_{0}^{-} \leq w$, induction shows that $u_{n}^{-} \leq w$ for all $n$. In particular,

$$
\liminf _{x \rightarrow-\infty} w(c ; x) \geq u_{n}^{-}(-\infty) \text { for all } n .
$$

For any fixed $n, u_{n}^{-}(x-z)$ converges to the constant $u_{n}^{-}(-\infty)$ uniformly on bounded intervals as $z \rightarrow-\infty$. Therefore,

$$
u_{n+1}(-\infty)=f^{-}\left(u_{n}^{-}(-\infty)\right) .
$$

Since $0<u_{0}(-\infty)=\gamma \alpha<\sigma$, and the $u_{n}^{-}(-\infty)$ are nondecreasing, they must converge to the nearest solution of the equation, $u=f^{-}(u)$. By definition, this solution is $\sigma$. Thus we have shown that $\liminf _{x \rightarrow-\infty} w(c ; x) \geq \sigma$.

It only remains to prove that if there is a solution $w(c ; x)$ of the traveling wave equation $w=Q_{c}[w]$ with $w(c, \infty)=0$ and $\liminf _{x \rightarrow-\infty} w(c ; x)>0$, then $c \geq c^{*}$. Suppose there is such a solution, and choose a nonincreasing function $v_{0}^{-}(x)$ such that $v_{0}^{-}(x) \leq w(c ; x), 0<v_{0}^{-}(-\infty)<\sigma$, and $v_{0}^{-}(x)=0$ for $x \geq 0$. Define $v_{n}^{-}$by the recursion $v_{n+1}^{-}=Q^{-}\left[v_{n}^{-}\right]$. Induction shows that $v_{n}^{-}(x) \leq w(c ; x-n c)$, so that for any positive $\epsilon$

$$
\lim _{n \rightarrow \infty}\left\{\sup _{x \geq(c+\epsilon) n} v_{n}^{-}(x)\right\} \leq \lim _{n \rightarrow \infty} w(c ; n \epsilon)=0 .
$$


The definition of the spreading speed $c^{*}$ of the recursion for $v^{-}$and the fact that $\epsilon$ is arbitrarily small show that $c^{*} \leq c$, and the proof of Theorem 4.1 is complete.

\section{Numerical simulations.}

In this section we present some approximations to traveling waves of the recursion (1.1) with the Laplace kernel

$$
k(|x-y|)=100 e^{-200|x-y|}
$$

and the Ricker recruitment function

$$
f(u)=u e^{r-u} .
$$

This function is increasing for $0 \leq u \leq 1$, and decreasing for $u \geq 1$. It is easily checked that the Hypotheses 2.1 are satisfied if and only if $r>0$, and that the positive solution of the equilibrium equation $u=f(u)$ is

$$
u^{*}=r
$$

Moreover,

$$
f^{+}(u)=\left\{\begin{array}{l}
f(u) \text { for } u \leq 1 \\
e^{r-1} \text { for } u \geq 1
\end{array}\right.
$$

so that

$$
\alpha=\left\{\begin{array}{l}
r \text { for } 0<r \leq 1 \\
e^{r-1} \text { for } r \geq 1
\end{array}\right.
$$

while

$$
f^{-}(u)=\min \left\{f(u), e^{2 r-1-e^{r-1}}\right\}
$$

so that

$$
\sigma=\left\{\begin{array}{l}
r \text { for } 0<r \leq 1 \\
e^{2 r-1-e^{r-1}} \text { for } r \geq 1
\end{array}\right.
$$

The following figures show approximations to the graphs of $w(x) / r$ where $w(x-n c)$ is a traveling wave of speed $c$ of (1.1), for several choices of $r$ and $c$. These graphs were obtained by an iterative process to find a fixed point of the operator $Q_{c}[u]$ defined in (4.1). The process uses the fact that a traveling wave of speed $c>c^{*}$ of (1.1) behaves like multiple of $e^{-\mu x}$ at infinity, where $\mu$ is the smaller root of the equation $(1 / \mu) \ln \left[f^{\prime}(0) K(\mu)\right]=$ $(1 / \mu) \ln \left[e^{r} /\left\{1-(\mu / 200)^{2}\right\}\right]=c$. A sufficiently small positive number $\mu$ was 
chosen, and $c$ was determined from this formula. The initial function $u_{0}$ was taken to be of the form $u_{0}=0.5 e^{-\mu(x-5)}$. For each $n, u_{n+1}$ was obtained from $u_{n}$ by setting it equal to a numerical approximation of $Q_{c}\left[u_{n}\right]$ for $x \leq 15-c$ and equating it to $.05 e^{-\mu(x-5)}$ for $x>15-c$. The numerical approximation to $Q\left[u_{n}\right]$ was based on the FFT algorithm of [1] with 32769 grid points. Because the positive equilibrium state is $u \equiv r$, we have graphed the normalized function $u_{100} / r$ for various values of $r$. These functions can be expected to be good approximations to $w(x) / r$.

When $r \leq 1$, we have $\alpha \leq 1$, so that $f$ is increasing on the range $0 \leq u \leq$ $\alpha$. In this case, it is easily seen that $f^{+}=f^{-}=f$ for $0 \leq u \leq \alpha=\sigma=u^{*}=$ $r$. Hence $w=w^{+}=w^{-}$is nonincreasing and has the limits $r$ at $-\infty$ and 0 at $+\infty$.

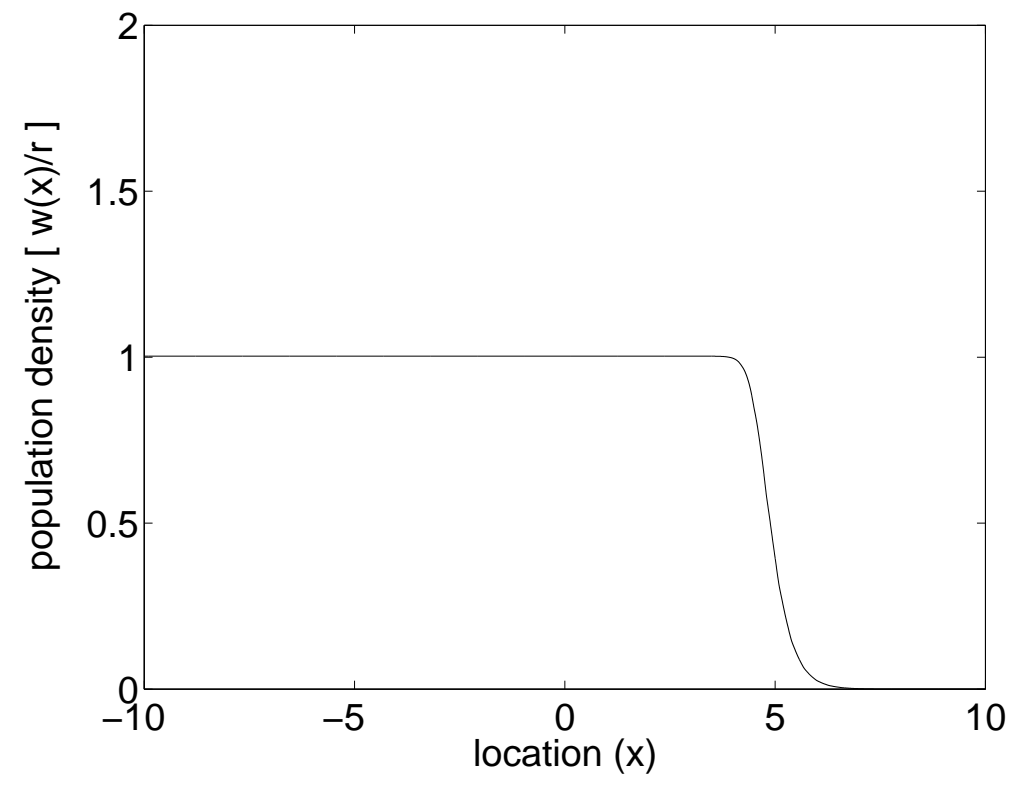

Figure 5.1

Figure 5.1 shows an approximation to the graph of $w(x) / r$ with $r=0.9$ and $c=0.30008$.

The proof of Lemma 3.10 of Thieme's paper [10] with a slightly adapted version of Lemma 3.10 there shows that if $u_{n}$ is a solution of (1.1) with $u_{0} \leq \alpha=e^{r-1}$, if $r<2$ so that the equilibrium $u \equiv r$ is stable, and if $0<c_{1}<c^{*}$, then

$$
\lim _{n \rightarrow \infty} \max _{|x| \leq n c_{1}}\left|r-u_{n}(x)\right|=0
$$

(Note that, as is the case in our example, $k(x)$ is assumed to be even in this Theorem, so that the leftward and rightward spreading speeds are both 
equal to $c^{*}$.) The definition of a traveling wave shows that the sequence $u_{n}(x)=w(x-n c)$ satisfies (1.1). Therefore, for any positive $\epsilon$ there is an $N_{\epsilon}$ such that $|r-w(x)| \leq \epsilon$ when $-\left(c+c_{1}\right) n \leq x \leq-n\left(c-c_{1}\right)$ and $n \geq N_{\epsilon}$. Because $c_{1}<c^{*} \leq c$, we have $c-c_{1}>0$. If $N_{\epsilon}$ is also chosen so large that $\left(2 N_{\epsilon}+1\right) c_{1}>c$, the above intervals for $n$ and $n+1$ overlap when $n \geq N_{\epsilon}$, and we conclude that $|r-w(x)| \leq \epsilon$ for all $x \leq-\left(c-c_{1}\right) N_{\epsilon}$. Since $\epsilon$ is arbitrary, we have shown that

$$
\lim _{x \rightarrow-\infty} w(x)=r \text { when } r<2
$$

That is, as long as $r<2$, the wave $w$ has the limit $r$ at $-\infty$.

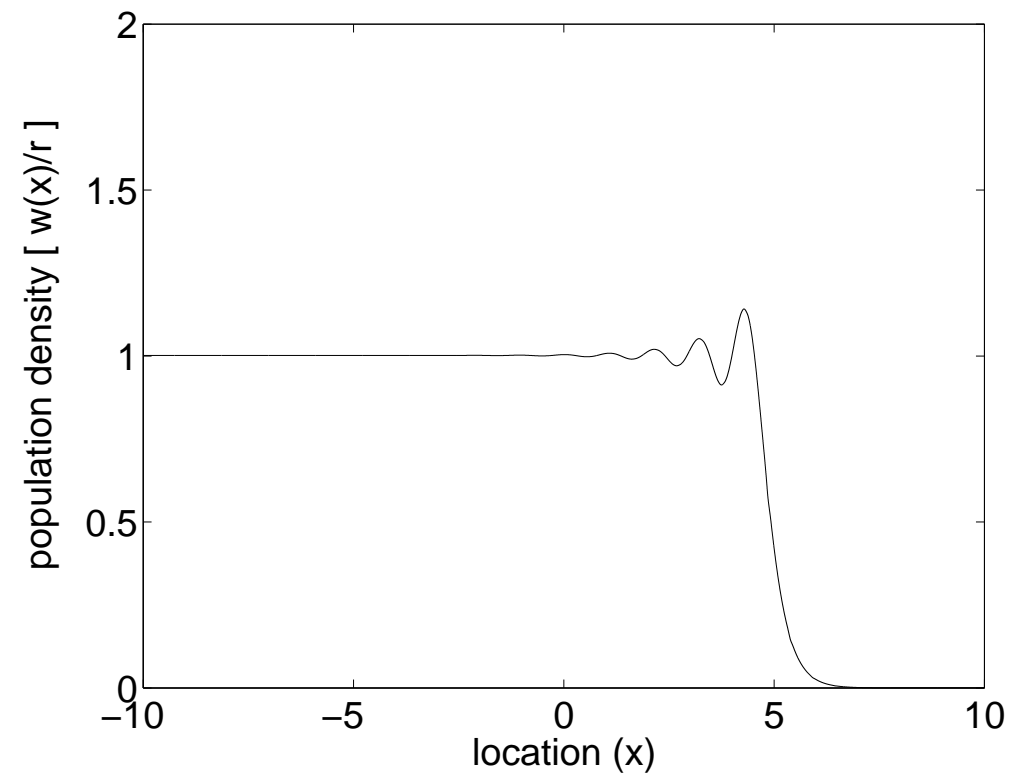

Figure 5.2

Figure 5.2 shows an approximation to the graph of $w(x) / r$ when $r=1.6$ and $c=0.5334$. We see that the graph oscillates about the value 1 , and that the oscillations are damped out for large negative $x$.

When $r>2$, the equilibrium solution $u_{n}=r$ is unstable. 


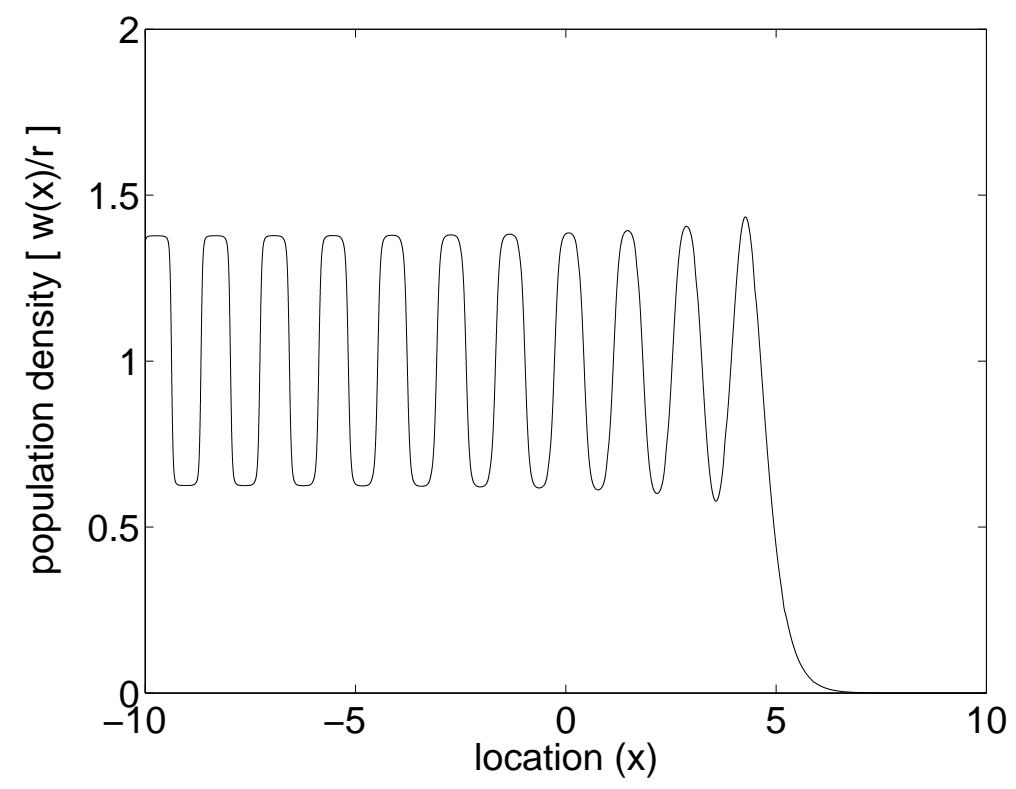

Figure 5.3

Figure 5.3 shows an approximation to the graph of $w(x) / r$ for $r=2.1$ and $c=.70008$. We note that the wave seems to continue oscillating all the way to $-\infty$. It appears, in fact, that the oscillations for large negative $x$ are periodic of period $2 c$, but we have not been able to prove this. As $r$ becomes larger, the periods in the tail can be expected to become shorter, so that the tail will eventually look chaotic.

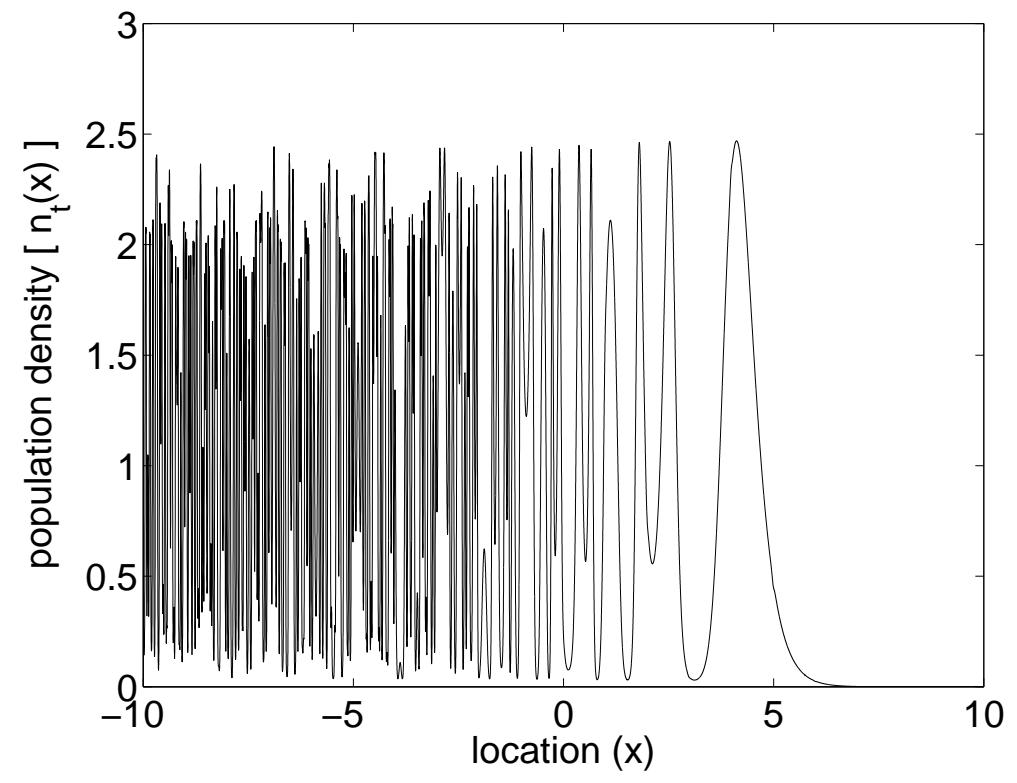

Figure 5.4. 
This behavior is illustrated by Figure 5.4, which shows the approximate wave of speed 1.0001 when $r=3$.

\section{Discussion.}

We have shown that for a class of integral recursion models with overcompensation, the asymptotic spreading speed can be characterized as the smallest speed of a natural class of non-constant traveling waves. When $k(x)$ is the Laplace kernel $(\beta / 2) e^{-\beta|x|}$, M. Kot [6] has predicted the existence of such traveling waves by means of a heuristic argument, and has asked other researchers to come up with a proof. Our Theorem 4.1 provides such a proof.

In the case of the logistic recruitment function $f(u)=u[1+r(1-u)]$ with $0<r<3$, M. Kot's simulations seem to indicate that for $r<2$, including some values at which the wave is not monotone, the solution of an initial value problem converges "in shape" to the traveling wave of speed $c^{*}$. However, simulations in [6], and unpublished simulations for the Ricker function by N. Shigesada and K. Kawasaki seem to show that when the parameter is slightly larger than the value at which the constant equilibrium becomes unstable, the solution of the initial value problem settles down "in shape", not to a time-independent solution, but to a solution which is periodic of period 2 in $n$, and that the period grows as stable spatially independent cycles arise with increasing $r$. Such solutions cannot converge to a steady wave of the kind we have constructed. However, Kot conjectured that the solutions which approach a $k$-cycle will converge in shape to a traveling wave of speed $k c^{*}$ of the recursion $u_{(n+1) k}=Q^{k}\left[u_{n k}\right]$, with $Q^{k}$ the $k$ th iterate of the operator $Q$. We know of no way to prove this conjecture.

The present paper has only treated the recursion (1.1) for a one-dimensional habitat. However, the traveling wave solution $w(\boldsymbol{\xi} \cdot \mathbf{x}-n c)$ of speed $c$ in the direction of the analogous recursion in two or more space dimensions is a solution of a recursion of the form (1.1), where the kernel $k$ depends on $c$ and $\boldsymbol{\xi}$. (See, e.g., [13].) Therefore, Theorem 4.1 of the present paper still gives the result that such a traveling wave exists if and only if $c \geq c^{*}(\boldsymbol{\xi})$, where $c^{*}(\boldsymbol{\xi})$ is the spreading speed defined in [13] for the operator with the nonmonotone function $f$ replaced by $f^{+}$.

In [9] and [10] H. Thieme considered an integral equation model for the population density $u(t, x)$ of mature individuals near $x$ at time $t$, which can 
be written in the form

$$
u(t, x)=a_{0}(t, x)+\int_{0}^{t-t_{0}} \int_{-\infty}^{\infty} k(s, y) f(u(t-s, x-y)) d y d s .
$$

Here $t_{0}$ is an initial time, $a_{0}(t, x)$ is the (predictable) density of mature individuals which were born before the initial time, $f$ is a fecundity function, and $k(s, y)$ represents the density of individuals which have survived and moved by a distance $y$ during a time $s$ after birth. In [3] O. Diekmann considered an epidemic model which also has the form (6.1), and showed that one can define a traveling wave of this equation in the following manner: Take a limit as the initial time $t_{0}$ recedes to $-\infty$, and $a_{0}$ becomes 0 , and look for a solution of the form $u(t, x)=w(x-c t)$ of the resulting equation. If we define the variables $X=x-c t$ and $Y=y-c s$, the equation for $w$ takes the form

$$
w(X)=\int_{-\infty}^{\infty} k_{c}(Y) f(w(X-Y)) d Y
$$

where we have defined the one-dimensional kernel

$$
k_{c}(Y):=\int_{0}^{\infty} k(s, Y+c s) d s .
$$

We note that the equation for $w$ says that $w$ is an equilibrium, that is, a traveling wave of speed 0 , of the recursion (1.1) with the migration kernel $k_{c}$. As long as $f(u)$ is nondecreasing, as it is in [3], this wave exists if and only if the spreading speed of this recursion is nonpositive. The formula (3.1) and the definition of $k_{c}$ show that this condition is equivalent to the inequality

$$
\int_{-\infty}^{\infty} \int_{0}^{\infty} e^{\mu(y-c s)} k(s, y) d s d y \leq 1 \text { for all } \mu>0 .
$$

Diekmann and Thieme independently found that the spreading speed of the equation (6.1) is the infimum of the numbers $c$ such that this inequality is valid. Diekmann then concluded that for his equation, for which $f$ is nondecreasing there are traveling waves for all $c$ which are at least equal to this spreading speed. The same conclusion holds for the more general but nondecreasing functions $f$ treated in [9]. For the functions with overcompensation treated in [10], on can obtain the same conclusion by using Theorem 4.1, provided the kernel $k_{c}$ and the fecundity $f$ satisfy the Hypotheses 2.1.

It may well be true that the same idea can be applied to the more general model of H. R. Thieme and X. Q. Zhao in [11], in which the product function $k(s, y) f(u(t-s, x-y))$ in $(6.1)$ is replaced by a function $F(u(t-s, x-y), s, y)$, 
with the function $F(v, s, y)$ subject to suitable hypotheses. The existence of a spreading speed is established under hypotheses similar to those of [10], but the existence of a traveling wave is only proved under the additional assumption that $F(v, s, y)$ is nondecreasing in $v$. The traveling wave satisfies the equation (6.2) with the product $m_{c} f$ replaced by a function $F_{c}(v, Y)$. The proof of Theorem 4.1 should establish the existence of waves under weaker hypotheses on $F$.

\section{References}

[1] M. Anderson, Properties of some density-dependent integrodifference equation population models. Mathematical Biosciences, 104:135-157, 1991.

[2] R. Courant and D. Hilbert, Methods of Mathematical Physics II. Interscience, New York, 1962.

[3] O. Diekmann, Thresholds and travelling waves for the geographical spread of an infection. J. of Math. Biol. 6:109-130, 1978.

[4] O. Diekmann, Run for your life. J. of Diff. Eq. 33: 58-73, 1979.

[5] A. Kolmogorov, Petrovsky and N.I. Piscounov, Étude de l'équation de la diffusion avec croissance de la quantité de matière et son application a un problème biologique. Bull. Moscow Univ. Math. Mech., 6(1):1-26, 1937.

[6] M. Kot, Discrete-time travelling waves: Ecological Examples. J. of Math. Biol., 30: 413-436, 1992.

[7] M. Kot, M. A. Lewis and P. van den Driessche, Dispersal data and the spread of invading organisms. Ecology, 77: 2027-2042, 1996. 
[8] R. M. May and G. F. Oster, Period doubling and the onset of turbulence: an analytic estimate of the Feigenbaum ratio. Physics Letters, 78A:1-3, 1980 .

[9] H. R. Thieme, Asymptotic estimates of the solutions of nonlinear integral equations and asymptotic speeds for spread of populations. J. für reine u. angew. Math.306: 94-121, 1979. ü

[10] H. R. Thieme, Density-Dependent Regulation of Spatially Distributed Populations and their Asymptotic speed of Spread. J. of Math. Biol., 8: 173-187 1979 .

[11] H. R. Thieme and X. Q. Zhao, Asymptotic speeds of spread and traveling waves for integral equations and delayed reaction-diffusion models. Journal of Differential Equations 195: 430-470, 2003

[12] H. F. Weinberger, Asymptotic behavior of a model in population genetics. In Nonlinear Partial Differential Equations and Applications, ed. J. M. Chadam Lecture Notes in Mathematics, Volume 648, pages 47-96. Springer-Verlag, Berlin, 1978.

[13] H. F. Weinberger, Long-time behavior of a class of biological models. SIAM J. Math. Anal., 13: 353-396, 1982. 